\title{
A narrative review of hybrid ablation for persistent and longstanding persistent atrial fibrillation
}

\author{
Ali Khoynezhad, Nikhil Warrier, Tiffany Worthington, Adrian Shandling \\ MemorialCare Heart and Vascular Institute, Long Beach, CA, USA \\ Contributions: (I) Conception and design: A Khoynezhad; (II) Administrative support: A Khoynezhad, T Worthington; (III) Provision of study \\ materials or patients: None; (IV) Collection and assembly of data: None; (V) Data analysis and interpretation: None; (VI) Manuscript writing: All \\ authors; (VII) Final approval of manuscript: All authors. \\ Correspondence to: Ali Khoynezhad, MD, PhD. Department of Cardiovascular Surgery, MemorialCare Heart and Vascular Institute, 2801 Atlantic \\ Ave, Long Beach, CA 90806, USA. Email: akhoynezhad@memorialcare.org.
}

\begin{abstract}
Atrial fibrillation is the most common sustained arrhythmia and is characterized by rapid and irregular atrial activation with loss of atrial contraction. There has been a significant evolution of treatments over the past 30 years. Initially, cardiac surgeons developed approaches via sternotomy with superior efficacy, however early iterations of the procedure were associated with prolonged recovery time and frequent need for pacemaker placement. The current surgical approach to the maze procedure via sternotomy yields excellent efficacy and is a Class 1 recommendation for patients with atrial fibrillation undergoing a concomitant procedure. Several years following the initial development of the surgical maze procedure, cardiac electrophysiologists developed less invasive, however less efficacious catheter ablation options by percutaneous approach. Both the surgical and transcatheter approaches have their advantages and disadvantages with varying risks of complications and efficacy. Through the combination of expertise of cardiac surgeons paired with the electrophysiology team, a hybrid ablation procedure has been developed offering an increased efficacy with a less-invasive approach than the current gold standard treatment of Coxmaze IV procedure. This review will discuss the hybrid ablation procedure, review recent associated clinical trials, and discuss advantages and challenges associated with this multidisciplinary approach for management of patients with AF.
\end{abstract}

Keywords: Atrial fibrillation; cardiac surgical procedures; electrophysiology

Submitted Jan 14, 2021. Accepted for publication Apr 26, 2021.

doi: 10.21037/atm-21-196

View this article at: http://dx.doi.org/10.21037/atm-21-196

\section{Introduction}

On September $25^{\text {th }}$, 1987, Dr. James Cox performed the first Cox-maze I procedure for the treatment of atrial fibrillation (1). The original procedure involved the cutting and sewing of areas on the heart, which had been previously identified through animal models to be sources for macro re-entrant circuits responsible for the propagation of AF (2). The procedure was subsequently successful in restoring sinus rhythm for several patients, however there was some left atrial dysfunction necessitating pacemaker placement as well as inability to accelerate rate in response to exertional activities (1). Further iterations to the procedure over the years led to the development of the Cox-maze III, which was associated with a $93 \%$ freedom from arrhythmia without antiarrhythmic medication (1). Despite the success of the Cox-maze III procedure, due to the technical complexity and prolonged cardiopulmonary bypass time required of performing the cut and sew lesion set, it was seldom performed. In 1999, the first Cox-maze IV procedure was performed, which included the lesion set presented in the Cox-maze III, however performed with cryothermal ablation to achieve the transmural lesions. The volume of surgical maze procedures remained low 
Table 1 Current HRS guideline recommendations (5)

\begin{tabular}{ll}
\hline $\begin{array}{l}\text { Indications for stand-alone and hybrid surgical } \\
\text { ablation of atrial fibrillation }\end{array}$ & Classification \\
\hline $\begin{array}{l}\text { Symptomatic AF refractory or intolerant } \\
\text { to at least one Class I or III antiarrhythmic } \\
\text { medication }\end{array}$ & $\begin{array}{l}\text { Persistent: Stand-alone surgical ablation is reasonable for } \\
\text { patients who have failed one or more attempts at catheter } \\
\text { ablation and also for those patients who prefer a surgical } \\
\text { approach after review of the relative safety and efficacy of } \\
\text { catheter ablation versus a stand-alone surgical approach. }\end{array}$ \\
& $\begin{array}{l}\text { Long-standing persistent: Stand-alone surgical ablation } \\
\text { is reasonable for patients who have failed one or more } \\
\text { attempts at catheter ablation and also for those patients } \\
\text { who prefer a surgical approach after review of the relative } \\
\text { safety and efficacy of catheter ablation versus a } \\
\text { stand-alone surgical approach. }\end{array}$ \\
\hline
\end{tabular}

initially, with STS Database reporting 3,987 AF procedures in 2004 which rose dramatically the following year to 12,737 procedures performed (3). Since then, the surgical management of $\mathrm{AF}$ has continued to rise, with improvements in ablation technology and experience minimizing the associated morbidity and increasing efficacy (4). Today, surgical ablation is a Class I recommendation for patients with AF undergoing concomitant open surgery, Class IIa for patients with AF undergoing closed surgery (CABG or AVR) (4) (Table 1).

Catheter ablation was first described in 1998 and continued to gain traction into the early 2000s (5). Initial attempts with transcatheter linear radiofrequency ablation in the right and left atria sought to replicate surgical procedures in patients (6). As pulmonary veins were identified as common triggers for patients with paroxysmal $\mathrm{AF}$, increased attention was spent on developing catheterbased techniques to achieve pulmonary vein isolation (5). Targeting these ectopic pulmonary vein triggers led to high incidence of pulmonary vein stenosis (7). Soon thereafter, pulmonary vein antral isolation using a non-fluoroscopic 3D electroanatomic navigation system was developed to create radiofrequency lesions around the pulmonary vein ostia (8). The antral approach avoids ablating inside the PVs, so that pulmonary vein stenosis incidence is reduced.

This now represents the primary ablation strategy for catheter-based ablation therapy of both paroxysmal and persistent AF (5). The majority of ablation procedures performed for AF are catheter based, with a growth of $430 \%$ between 2006 and 2015 (9). Although catheter ablation is associated with lower morbidity than surgical maze procedures, the efficacy is also reduced. Patients who undergo catheter ablation often require repeat procedures to achieve maintained sinus rhythm, especially in persistent and longstanding persistent $\mathrm{AF}$.

Adjunctive ablation lesion sets can be performed in these patients to address non pulmonary vein triggers that potentially contribute to the initiation and maintenance of AF (10). This includes addressing triggers originating from the superior vena cava, inferior vena cava, left atrial appendage, coronary sinus ostium, the vein of Marshall and posterior wall as well as complex fractionated atrial electrogram ablation and rotor ablation. Many of these strategies lack data from large randomized controlled trials.

For patients with symptomatic paroxysmal AF refractory to antiarrhythmic medication, catheter ablation currently has a Class IA recommendation (5). For patients with persistent and longstanding persistent AF (greater than one year), the recommendations are IIa, and IIb respectively (5). For patients with persistent or long-standing persistent AF, posterior wall isolation and ablation of non $\mathrm{PV}$ triggers also carry a Class IIb recommendation (5).

We present the article in accordance with the Narrative Review reporting checklist (available at http://dx.doi. org/10.21037/atm-21-196).

\section{Cox-maze IV procedure}

The goal of the maze procedure regardless of approach or lesion set is to create transmural ablation lines which prevent the conduction of re-entry circuits responsible for the propagation of atrial fibrillation. The Cox-maze IV procedure can be performed via sternotomy or right antero-lateral thoracotomy. Pulmonary vein isolation is 
achieved using bipolar radiofrequency clamps. Right atrial lesions include an ablation along the free edge of the right atrium down the aortic side of the right atrial appendage. An ablation line is extended from superior to inferior vena cavae. An endocardial ablation line is created using a cryoprobe extending from the right atrial free wall to the tricuspid valve annulus. In the left atrium, a "box lesion" is achieved by creating roof and floor lines connecting the superior and inferior pulmonary veins. A lesion extending from the left atrial appendage to the pulmonary vein as well as one extending to the mitral isthmus is created. The left atrial appendage, a known potential trigger for $\mathrm{AF}$ is also excluded (11).

\section{Thoracoscopic hybrid maze}

Hybrid ablation procedures for the treatment of AF have been proposed in several single-center trials, with promising results, however there has been a lack of uniformity in lesion sets, timing of surgical ablation (SA) versus catheter ablation (CA), rigor of patient follow-up, type of energy used, and management approaches to left atrial appendage.

The goal of hybrid AF ablation is to achieve the Cox-maze IV lesion set through a minimally invasive thoracoscopic approach followed by catheter ablation, thereby obviating the need for sternotomy and cardiopulmonary bypass while providing the patient with the best possible outcomes for management of AF. With the direct visualization afforded through endoscopic access to the thoracic cavity, the surgeon can perform pulmonary vein isolation utilizing a bipolar radiofrequency clamp, which completely surrounds the antrum of the pulmonary veins in a single movement. Pulmonary vein isolation is the cornerstone of $\mathrm{AF}$ ablation in most patients. Through the epicardial approach, the surgeon can avoid critical structures that can be injured during catheter ablation including the phrenic nerve, pulmonary vein orifices, and esophagus. Additionally, the ganglionic plexi and ligament of Marshall can be ablated under direct visualization and left atrial appendage can be excluded during the epicardial procedure. Following the epicardial procedure, the electrophysiologist performs completion lesions from an endocardial approach. Gaps in the epicardial "box lesions" can be addressed, pulmonary vein isolation can be verified, and ablation to sites not accessible through the epicardial approach can be achieved including the mitral isthmus lesion which lies close to the left circumflex artery as well as the coronary sinus and the cavotricuspid isthmus in the right atrium. In patients with increased epicardial fat, transmurality of epicardial lesions can be challenging, and better achieved through the endocardial approach. Following the completion of the Cox-maze IV lesion set pattern, the electrophysiologist has the opportunity to evaluate for additional AF trigger foci.

\section{Advantages and challenges}

Through a team-based approach to ablation, the electrophysiologist can provide feedback to the surgeon regarding transmurality of epicardial lesions as well as gaps in the ablation lines. There are limitations to an epicardial only lesion set; epicardial fat affects the transmurality of lesions and the heat sink effect of circulating left atrial blood limits depth of ablation. In endocardial only lesion sets, there is a risk of collateral damage to surrounding structures. By combining the strengths of both approaches, effectiveness is improved and the limitations are reduced. Timing of the epicardial and endocardial procedures has been variable in studies to date when it comes to concomitant or staged completion. There are advantages and disadvantages to performing the procedures concomitantly versus a staged approach. With the concomitant procedure, there is a decreased associated cost as well as a single hospitalization for the patient. It can be challenging to align the schedules of the surgeon and electrophysiologist for the concomitant approach. In addition, edema resulting from the surgical ablation may show block initially, but when edema has subsided these areas may reveal incomplete lesions. During a staged procedure, there is time to allow for fibrous tissue formation of the ablation lines allowing for a clear picture of gaps in lesions and to allow for closure of those gaps. To date, there is insufficient data to determine whether a single or staged approach would be superior (5). Recommendations have been made to delay the endocardial procedure for 1 to 3 months following the epicardial ablation (12) (Table 2).

\section{Outcomes}

There is a range of reported 1-year freedom from $\mathrm{AF}$ for a hybrid ablation for AF (Table 3). One meta-analysis reported $71 \%$ for hybrid ablation versus $87 \%$ for Cox-maze (19). Another meta-analysis reported successful freedom from atrial fibrillation following hybrid $\mathrm{AF}$ procedures ranging from $36.8 \%$ to $92 \%$ (20). These studies are plagued by lack of uniformity in approaches, lesion sets, timing to CA, and follow-up protocols. Recent studies over the past 2 years continue to illustrate the variability in these factors (Table 4). 
Table 2 Rationale of hybrid ablation of atrial fibrillation

Creation of completed lines

Surgical approach may be more complete in making transmural ablation lines

Ablation tools are designed for making lines

Smooth epicardial surface ideal for surgical tools

Visual imaging reveals the atrial surface, ablation lines, and gaps in lesions

Catheter ablation may be most effective in targeting specific lesions

Catheter ablation is designed to create point lesions

Catheter can slip off endocardial ridges or trabeculations, thus breaking up lines

Even with ultrasound imaging, assessing continuing of endocardial lesions may be difficult

Complimentary nature of epicardial and endocardial ablation

Epicardial ablation

Heat sink of the circulating blood in the atrial chamber limits depth

Epicardial lesions may be limited by fat

Depth of ablation lesions may be insufficient

May fail to penetrate the endocardium

Endocardial ablation

Creating transmural lesions may be difficult

Endocardial ablation may result in collateral damage to epicardial structures

Role of mapping

Epicardial mapping may be limited

Constrained by pericardial reflections

Absence of sophisticated tools and mapping systems designed for epicardial use

Epicardial fat may limit mapping

Endocardial mapping

Extensive experience in mapping

Large range of tools and technology

Formally trained

Mature enabling technology

Role of mapping

Unique targets

Surgical epicardial ablation

Full division of ligament of Marshall

LAA removal

Targeted ganglionic plexi ablation

Safer superior vena cava isolation

Table 2 (continued) 
Table 2 (continued)

Transcatheter endocardial ablation

More effective cavotricuspid isthmus line

Atrial flutter and atrial tachycardia ablation

Coronary sinus ablation

Map for flutter

Mapping techniques, such as FIRM or CFAE

CFAE, complex fractionated atrial electrograms; FIRM, focal impulse and rotor modulation; LAA, left atrial appendage. From Khoynezhad et al. (13).

Table 3 Summary of recent studies evaluating hybrid atrial fibrillation ablation

\begin{tabular}{lccccccc}
\hline First author & Year & Patients, $\mathrm{n}$ & P-LSP, $\%$ & Access & Timing & Mortality, \% & Complications, \% \\
\hline Haywood (14) & 2020 & 175 & 100 & R-Thor & Staged & 0.6 & 20 \\
Choi (15) & 2020 & 23 & 100 & B-Thor & Staged & 0 & 20 \\
de Asmundis (16) & 2019 & 51 & 100 & L-Thor & Staged & 0 & 4 \\
Al-Jazairi (17) & 2019 & 50 & 90 & B-Thor & Concomitant & 0 & 14 \\
Pojar (18) & 2018 & 65 & 54 & B-Thor & Staged & 0 & 0 \\
\hline
\end{tabular}

AF, atrial fibrillation; B-Thor, bilateral thoracoscopic; P-LSP, persistent and long standing persistent; R-Thor, right thoracoscopic.

Table 4 Current clinical trials for hybrid ablation

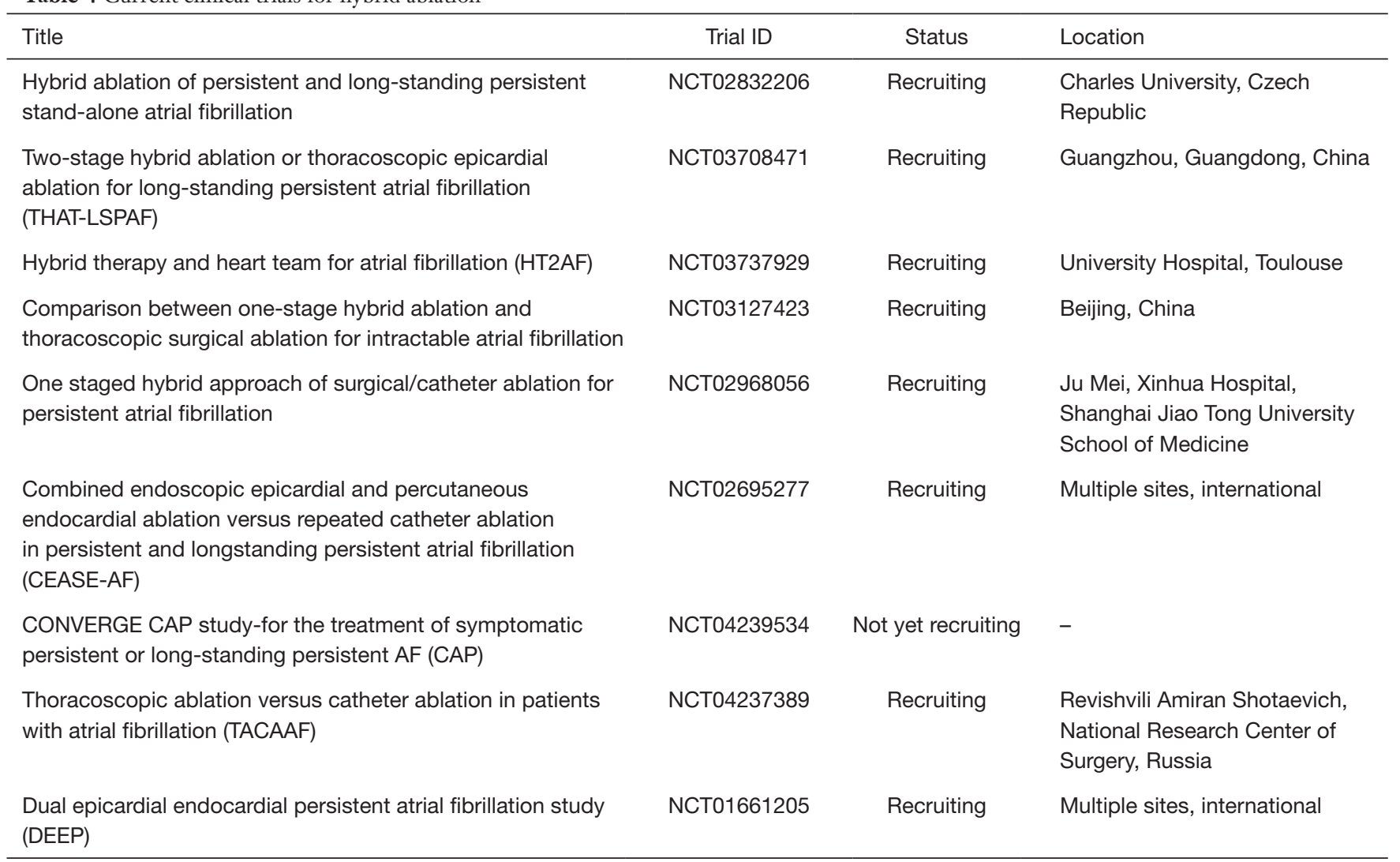




\section{Negative factors for outcomes}

Some factors have been recognized as predictors for failure of ablation and should be used in the screening of patients when considering whether to proceed with a surgical or catheter ablation. These risk factors include increased left atrial size, advanced age, and extended duration of AF. In surgical ablation, anatomic locations are used to determine placement of lesions, where in catheter ablation there is the added benefit of mapping to aid in lesion creation. For patients with substantial epicardial fat, achieving transmurality of ablation lines can be challenging from the surgical approach leading to incomplete lesions. Additionally, there are some procedure-based limitations including lesion set pattern types. If a lesion set is restricted to only the left atrium, there are right-sided foci that may continue to trigger AF. The ablation energy source selected and experience of the operator are also important factors in the efficacy of surgical AF ablation.

\section{Conclusions}

Atrial fibrillation management remains a challenge, especially in patients with persistent and longstanding persistent AF. Through a team-based approach, hybrid ablation of persistent and longstanding persistent AF utilizes the strengths of electrophysiology and surgery and offers an alternative management process to improve outcomes. While data from current trials show promising results in management of patients with non-paroxysmal atrial fibrillation, ongoing clinical trials will lead to standardization of the protocols with respect to lesions sets, timing of CA as well as follow up protocols.

\section{Acknowledgments}

Funding: None.

\section{Footnote}

Reporting Checklist: The authors have completed the Narrative Review reporting checklist. Available at http:// dx.doi.org/10.21037/atm-21-196

Conflicts of Interest: All authors have completed the ICMJE uniform disclosure form (available at: http://dx.doi. org/10.21037/atm-21-196). AK serves as an unpaid editorial board member of Annals of Translational Medicine from May
2019 to Apr 2021. The authors have no other conflicts of interest to declare.

Ethical Statement: The authors are accountable for all aspects of the work in ensuring that questions related to the accuracy or integrity of any part of the work are appropriately investigated and resolved.

Open Access Statement: This is an Open Access article distributed in accordance with the Creative Commons Attribution-NonCommercial-NoDerivs 4.0 International License (CC BY-NC-ND 4.0), which permits the noncommercial replication and distribution of the article with the strict proviso that no changes or edits are made and the original work is properly cited (including links to both the formal publication through the relevant DOI and the license). See: https://creativecommons.org/licenses/by-nc-nd/4.0/.

\section{References}

1. Cox JL, Schuessler RB, D'Agostino HJ Jr, et al. The surgical treatment of atrial fibrillation. III. Development of a definitive surgical procedure. J Thorac Cardiovasc Surg 1991;101:569-83.

2. Boineau JP, Schuessler RB, Mooney CR, et al. Natural and evoked atrial flutter due to circus movement in dogs. Role of abnormal atrial pathways, slow conduction, nonuniform refractory period distribution and premature beats. Am J Cardiol 1980;45:1167-81.

3. Gammie JS, Haddad M, Milford-Beland S, et al. Atrial fibrillation correction surgery: lessons from the Society of Thoracic Surgeons National Cardiac Database. Ann Thorac Surg 2008;85:909-14.

4. Lall SC, Melby SJ, Voeller RK, et al. The effect of ablation technology on surgical outcomes after the Cox-maze procedure: a propensity analysis. J Thorac Cardiovasc Surg 2007;133:389-96.

5. Ad N, Damiano RJ, Badhwar V, et al. Expert consensus guidelines: examining surgical ablation for atrial fibrillation. J Thorac Cardiovasc Surg 2017;153:133054.e1.

6. Haïssaguerre M, Jaïs P, Shah DC, et al. Spontaneous initiation of atrial fibrillation by ectopic beats originating in the pulmonary veins. N Engl J Med 1998;339:659-66.

7. Pappone C, Oreto G, Lamberti F, et al. Catheter ablation of paroxysmal atrial fibrillation using a 3D mapping system. Circulation 1999;100:1203-8.

8. Chen SA, Hsieh MH, Tai CT, et al. Initiation of 
atrial fibrillation by ectopic beats originating from the pulmonary veins: electrophysiological characteristics, pharmacological responses, and effects of radiofrequency ablation. Circulation 1999;100:1879-86.

9. Pappone C, Rosanio S, Oreto G, et al. Circumferential radiofrequency ablation of pulmonary vein ostia: A new anatomic approach for curing atrial fibrillation. Circulation 2000;102:2619-28.

10. Holmqvist F, Kesek M, Englund A, et al. A decade of catheter ablation of cardiac arrhythmias in Sweden: ablation practices and outcomes. Eur Heart J 2019;40:820-30.

11. Lin WS, Tai CT, Hsieh MH, et al. Catheter ablation of paroxysmal atrial fibrillation initiated by non-pulmonary vein ectopy. Circulation 2003;107:3176-83.

12. Di Biase L, Burkhardt JD, Mohanty P, et al. Left atrial appendage: an underrecognized trigger site of atrial fibrillation. Circulation 2010;122:109-18.

13. Khoynezhad A, Ellenbogen KA, Al-Atassi T, et al. Hybrid atrial fibrillation ablation: current status and a look ahead. Circulation 2017;10:e005263.

14. Haywood GA, Varini R, Osmancik P, et al. European Multicentre Experience of Staged Hybrid Atrial Fibrillation Ablation for the Treatment of Persistent and Longstanding Persistent Atrial Fibrillation. Int J Cardiol Heart Vasc 2020;26:100459.

Cite this article as: Khoynezhad A, Warrier N, Worthington T, Shandling A. A narrative review of hybrid ablation for persistent and longstanding persistent atrial fibrillation. Ann Transl Med 2021;9(11):947. doi: 10.21037/atm-21-196
15. Choi MS, On YK, Jeong DS, et al. Usefulness of Postprocedural Electrophysiological Confirmation Upon Totally Thoracoscopic Ablation in Persistent Atrial Fibrillation. Am J Cardiol 2020;125:1054-62.

16. de Asmundis C, Varnavas V, Sieira J, et al. Two-year follow-up of one-stage left unilateral thoracoscopic epicardial and transcatheter endocardial ablation for persistent and long-standing persistent atrial fibrillation. J Interv Card Electrophysiol 2020;58:333-43.

17. Al-Jazairi MI, Rienstra M, Klinkenberg TJ, et al. Hybrid Atrial Fibrillation Ablation in Patients with Persistent Atrial Fibrillation or Failed Catheter Ablation. Neth Heart J 2019;27:142-51.

18. Pojar M, Haman L, Parizek P, et al. Midterm Outcomes of Mini-Invasive Surgical and Hybrid Ablation of Atrial Fibrillation. Biomed Pap Med Fac Univ Palacky Olomouc Czech Repub 2019;163:233-40.

19. Choi MS, Jeong DS. Staged hybrid procedure in persistent atrial fibrillation: safety, efficacy, and atrial tachyarrhythmia. J Thorac Dis 2017;9:4844-8.

20. Je HG, Shuman DJ, Ad N. A systematic review of minimally invasive surgical treatment for atrial fibrillation: a comparison of the Cox-Maze procedure, beating-heart epicardial ablation, and the hybrid procedure on safety and efficacy. Eur J Cardiothorac Surg 2015;48:531-40; discussion 540-1. 